\title{
Influence of Moxifloxacin on Hepatic Redox Status and Plasma Biomarkers of Hepatotoxicity and Nephrotoxicity in Rat
}

\author{
Ayokanmi Ore and Ebenezer Tunde Olayinka \\ Biochemistry Unit, Department of Chemical Sciences, Ajayi Crowther University, PMB 1066, Oyo 211213, Oyo State, Nigeria \\ Correspondence should be addressed to Ayokanmi Ore; oreayokanmi@gmail.com
}

Received 31 July 2015; Accepted 20 September 2015

Academic Editor: Andrei Surguchov

Copyright ( 2015 A. Ore and E. T. Olayinka. This is an open access article distributed under the Creative Commons Attribution License, which permits unrestricted use, distribution, and reproduction in any medium, provided the original work is properly cited.

Moxifloxacin is a broad spectrum fluoroquinolone antibacterial agent. We examined the hepatic redox status and plasma biomarkers of nephrotoxicity and hepatotoxicity in rat following administration of moxifloxacin (MXF). Twenty-four Wistar rats, 180-200 g, were randomized into four groups (I-IV). Animals in group I (control) received $1 \mathrm{~mL}$ of distilled water, while animals in groups II, III, and IV received $1 \mathrm{~mL}$ each of MXF equivalent to $4 \mathrm{mg} / \mathrm{kg}$ b.w., $8 \mathrm{mg} / \mathrm{kg}$ b.w., and $16 \mathrm{mg} / \mathrm{kg}$ b.w., respectively. After seven days, plasma urea, bilirubin, and creatinine were significantly $(P<0.05)$ elevated in the MXF-treated animals. Activities of alkaline phosphatase, aspartate aminotransferase, and alanine aminotransferase were significantly increased in the plasma of MXFtreated animals compared to control. Also plasma total cholesterol, HDL-cholesterol, LDL-cholesterol, and triglycerides increased significantly in the MXF-treated groups relative to control. Moreover, MXF triggered a significant decrease in hepatic catalase, superoxide dismutase, and glutathione-S transferase activities. Likewise, MXF caused a decrease in the hepatic levels of glutathione and vitamin C. A significant increase in hepatic MDA content was also observed in the MXF-treated animals relative to control. Overall, our data suggest that the half-therapeutic, therapeutic, and twice the therapeutic dose of MXF induced nephrotoxicity, hepatotoxicity, and altered hepatic redox balance in rats.

\section{Introduction}

Moxifloxacin (MFX) is a fourth-generation synthetic fluoroquinolone antibacterial agent with a broad spectrum of bactericidal action. MXF possess enhanced activity against Gram-positive bacteria, most notably against penicillinsusceptible and penicillin-resistant strains of S. pneumoniae. It is available for oral and intravenous administration, respectively, as a once-daily $400 \mathrm{mg}$ antibiotic for the treatment of respiratory tract infections, chronic bronchitis, and acute bacterial sinusitis and in some cases pelvic inflammatory disease, complicated and uncomplicated skin and skin structure infections, complicated intra-abdominal infections, ocular bacterial keratitis, and community acquired pneumonia [13].

MXF like other quinolones have a bicyclic aromatic core with a carbon at position 8 and demonstrate an N-1 cyclopropyl moiety (Figure 1). Following oral administration,
MXF is well absorbed from the gastrointestinal tract with approximately $50 \%$ bound to serum proteins [4]. It binds weakly to plasma proteins and penetrates well into most tissue and fluid compartments. MXF is metabolized to an Nsulfate conjugate and an acyl glucuronide in humans [5]. Like other fluoroquinolones, MXF exhibit bactericidal activity by binding to bacterial topoisomerases II (DNA gyrase) and topoisomerase IV [6]. By binding to these enzymes, the fluoroquinolones interfere with DNA replication, repair, and transcription, resulting in bacterial death. The ability to target both enzymes has been promoted as a major advantage of the fluoroquinolones in preventing or delaying the emergence of resistance [7].

Most fluoroquinolones are known to be associated with some adverse effects on vital organs [8]. Previous reports suggest that free radical formation might play a role in the mechanism of some of these adverse effects [9]. Moreover, certain members of the fluoroquinolones are known to 


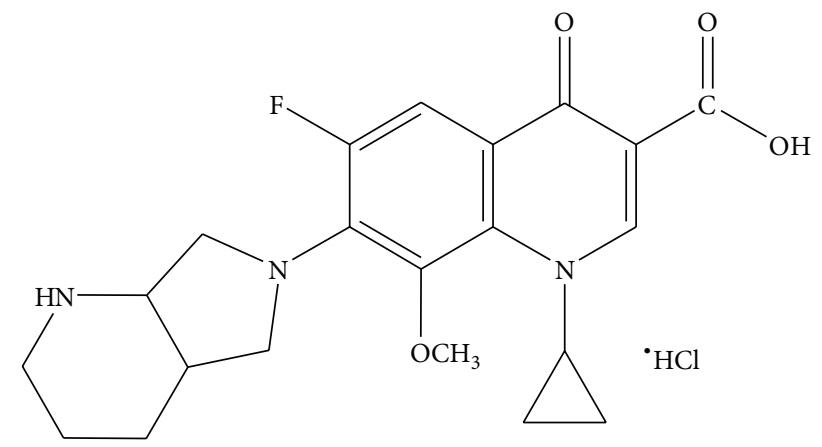

FIgURE 1: Moxifloxacin hydrochloride (1-cyclopropyl-7-[(S,S)-2,8diazabicyclo[4.3.0]non-8-yl]-6-fluoro-8-methoxy-1,4-dihydro-4oxo-3-quinoline carboxylic acid ${ }^{\bullet} \mathrm{HCl}$ ).

produce reactive oxygen species (ROS) in phagocytic cells $[10,11]$. Side effects such as hepatotoxicity [12], phototoxicity [13], and cartilage damage [11] may be related to generation of ROS [14] and weakening of enzymatic antioxidant defence mechanism in tissues [15].

The hepatocytes like most body cells are made up of antioxidant defence system comprising nonenzymatic antioxidants including glutathione, ascorbic acid, and tocopherol and enzymatic antioxidants such as catalase, superoxide dismutase, glutathione peroxidase, and glutathione reductase [16]. They are involved in protection against tissue injuries caused by free radicals and other ROS [16]. It has been suggested that many classes of antibiotics generate varying degrees of reactive oxygen species (ROS) that contribute to cell killing [17, 18]. However, exposure to elevated level of ROS may result in weakening of cellular antioxidant capacity thereby exposing the cell to excessive level of lipid peroxidation and ultimately tissue injury [19].

Our interest in the capacity of certain fluoroquinolones to generate ROS $[20,21]$ prompted us to study the effects of MFX on the hepatic biomarkers of oxidative stress in rat. There is little or no previous report on the effect of MXF on the hepatic antioxidant defence system as well as markers of nephrotoxicity and hepatotoxicity. Hence, this study was designed to assess the impact of MXF on hepatic redox status as well as biomarkers of nephrotoxicity and hepatotoxicity in rat model.

\section{Materials and Methods}

2.1. Chemicals and Reagents. Moxifloxacin hydrochloride (Avelox) was a product of Bayer Healthcare Pharmaceutics, Leverkusen, Germany. Glutathione (GSH), 1-chloro2,4-dinitrobenzene (CDNB), $5^{\prime}, 5^{\prime}$-dithiobis-2-nitrobenzoic acid (DTNB), thiobarbituric acid (TBA), epinephrine, and hydrogen peroxide were purchased from Sigma Chemical Company (London, UK). Assay kits for alanine aminotransferase (ALT), aspartate aminotransferase (AST), alkaline phosphatase (ALP), $\gamma$-glutamyl transferase ( $\gamma$-GT), urea, creatinine, bilirubin, total cholesterol, HDL-cholesterol, LDLcholesterol, and triglycerides were products of Randox Laboratories Ltd., Antrim, UK. All other chemicals and reagents used in this study were of analytical grade.

2.2. Animal Selection and Care. Twenty-four male albino rats (Wistar strain) weighing between $160 \mathrm{~g}$ and $180 \mathrm{~g}$ were used for this study. The animals were obtained from the animal holding unit of the Department of Chemical Sciences, Ajayi Crowther University, Oyo, Nigeria. The rats were acclimatised under laboratory conditions prior to the commencement of the study. The animals were housed in wire meshed cages maintained at standard conditions of temperature and humidity with an alternating light cycle $(12 \mathrm{hr}$ light/dark). They were fed with commercial pelletized diet (Ladokun Feeds, Ibadan, Nigeria) and supplied water ad libitum. The experimental protocol relating to animal handling conformed to the international guidelines on the Care and Use of Laboratory Animals [22].

2.3. Animal Grouping and Drug Administration. The rats were randomised into four experimental groups (I-IV) of 6 animals each. Group I (control) animals were administered distilled water. Animals in group II received MXF at a dose of $4 \mathrm{mg} / \mathrm{kg}$ body weight (b.w.); this is an equivalent of half of the therapeutic dose (MXF-1). Group III animals were administered $8 \mathrm{mg} / \mathrm{kg}$ b.w. MXF equivalent to the therapeutic dose used in the treatment of skin structure infection and community acquired pneumonia (MXF-2). Animals in group IV were administered MXF at $16 \mathrm{mg} / \mathrm{kg}$ b.w., equivalent to two times the therapeutic dose (MXF-3). The drug treatments lasted for 7 days.

2.4. Collection of Blood and Liver Samples. Blood samples were collected from each animal through retro orbitals plexus into heparinized tubes (Li heparin). Animals were thereafter euthanized and the liver was carefully excised from each animal for preparation of cytosolic fraction.

2.5. Preparation of Plasma and Cytosolic Fractions. Plasma was obtained by centrifugation of whole blood sample at $4000 \mathrm{rpm}$ for 5 minutes using a bench centrifuge (Analytika, Athens, Greece). The plasma obtained was stored at $-4^{\circ} \mathrm{C}$ for subsequent plasma assays. Liver samples obtained from each rat were blotted of blood stains, rinsed in ice-cold $1.15 \% \mathrm{KCl}$, and homogenized in 4 volumes of ice-cold $0.01 \mathrm{M}$ potassium phosphate buffer ( $\mathrm{pH}$ 7.4). The homogenates were centrifuged at $12,500 \mathrm{~g}$ for $15 \mathrm{~min}$ at $-4^{\circ} \mathrm{C}$ in a refrigerated centrifuge (Eppendorf, Stevenage, UK) and the supernatants, termed the postmitochondrial fractions (PMF), were aliquoted and used for subsequent biochemical assays.

\subsection{Determination of Plasma and Liver Protein Content.} Protein concentration in the plasma and liver homogenate was determined by the Biuret method of Gornall et al. [23] using bovine serum albumin as standard. 
2.7. Assay of Plasma Biomarkers of Renal Toxicity. Plasma urea and creatinine were determined with Randox diagnostic kits. Method for creatinine assays was based on colorimetric alkaline picrate methods of Jaffé [24] with creatinine-picrate complex measured at $492 \mathrm{~nm}$. Plasma urea determination was based on the Fenton reaction of Tietz [25] with the Diazine chromogen formed absorbing strongly at $540 \mathrm{~nm}$.

2.8. Assay of Plasma Biomarkers of Hepatotoxicity. Plasma total bilirubin (TBILI) determination was done using Randox diagnostic kits based on the dimethyl sulphoxide method by Tietz et al. [26]. The dimethyl sulphoxide forms a coloured compound with maximum absorption at $550 \mathrm{~nm}$. Plasma alkaline phosphatase (ALP), alanine aminotransferase (ALT), and aspartate aminotransferase (AST) activities were determined using Randox diagnostic kits. ALP activity was determined in accordance with the principles of Tietz et al. [26]. The p-nitrophenol formed by the hydrolysis of $\mathrm{p}$-nitrophenyl phosphate confers yellowish colour on the reaction mixture and its intensity can be monitored at $405 \mathrm{~nm}$ to give a measure of enzyme activity. Determination of plasma ALT and AST activities was based on the principle described by Reltman and Frankel [27]. ALT activity was measured by monitoring the concentration of pyruvate hydrazone formed with 2,4-dinitrophenylhydrazine at $546 \mathrm{~nm}$. AST activity was measured by monitoring the concentration of oxaloacetate hydrazone formed with 2,4-dinitrophenylhydrazine at $546 \mathrm{~nm}$.

2.9. Determination of Plasma Lipid Profiles. The plasma total cholesterol, HDL-cholesterol, LDL-cholesterol, and triglycerides were determined using Randox diagnostic kits and the determination was based on CHOD-PAD enzymatic colorimetric method of Trinder [28].

2.10. Assay for Nonenzymatic Antioxidants in the Liver. Hepatic reduced glutathione level was determined according to the method of Jollow et al. [29]. The chromophoric product resulting from the reaction of Ellman's reagent with the reduced glutathione, 2-nitro-5-thiobenzoic acid, possesses a molar absorption at $412 \mathrm{~nm}$ which was read in a spectrophotometer. Reduced GSH is proportional to the absorbance at $412 \mathrm{~nm}$. The ascorbic acid (AA) concentration was determined according to the method of Jagota and Dani [30]. AA in biological samples reacts with Folin's reagent, an oxidizing agent, to give a blue color which has its maximum absorption at $760 \mathrm{~nm}$.

2.11. Assay of Hepatic Antioxidant Enzymes. Hepatic glutathione-S-transferase (GST) activity was determined by the method described by Habig et al. [31] using 1-chloro2,4-dinitrobenzene (CDNB) as substrate. The procedure of Misra and Fridovich [32] was used for the determination of hepatic superoxide dismutase (SOD) activity by measuring the inhibition of autooxidation of epinephrine at $\mathrm{pH} 10.2$ and $30^{\circ} \mathrm{C}$. Hepatic catalase activity was determined by the method described by Sinha [33] based on the reduction of dichromate in acetic acid to chromic acetate when heated
TABLE 1: Influence of MXF on plasma biomarkers of renal toxicity in rat.

\begin{tabular}{lcc}
\hline Treatment groups & Urea $(\mathrm{mg} / \mathrm{dL})$ & Creatinine $(\mathrm{mg} / \mathrm{dL})$ \\
\hline Control & $41.2 \pm 3.8$ & $0.37 \pm 0.06$ \\
MXF-1 & $51.3 \pm 2.1(25 \%)^{*}$ & $0.54 \pm 0.03(46 \%)^{*}$ \\
MXF-2 & $55.5 \pm 3.5(35 \%)^{*}$ & $0.66 \pm 0.05(78 \%)^{*}$ \\
MXF-3 & $57.2 \pm 2.6(39 \%)^{*}$ & $0.88 \pm 0.07(137 \%)^{*}$ \\
\hline
\end{tabular}

Values represent the mean \pm SD of six replicates. ${ }^{*}$ Significantly different from control $(P<0.05)$; values in parenthesis represent $\%$ of increase.

in the presence of hydrogen peroxide $\left(\mathrm{H}_{2} \mathrm{O}_{2}\right)$. The chromic acetate produced is measured spectrophotometrically at $570 \mathrm{~nm}$

2.12. Assay of Hepatic Level of Lipid Peroxidation. The extent of lipid peroxidation (LPO) in the liver was estimated by the method of Varshney and Kale [34]. The method involved the reaction between malondialdehyde (MDA; product of lipid peroxidation) and thiobarbituric acid to yield a stable pink chromophore with maximum absorption at $532 \mathrm{~nm}$.

2.13. Statistical Analysis. Results were expressed as mean of 5 replicates \pm SD. Data obtained were subjected to one-way Analysis of Variance (ANOVA) followed by Duncan multiple range test for comparison between control and treated rats in all groups using SigmaPlot Statistical application package. $P$ values less than 0.05 were considered statistically significant.

\section{Results}

3.1. Influence of MXF on Plasma Biomarkers of Nephrotoxicity in Rat. Plasma levels of urea and creatinine have been considered suitable biomarkers of renal function. Table 1 represents the plasma levels of urea and creatinine in rats following administration of MXF. Plasma urea level increased significantly $(P<0.05)$ by $25 \%, 35 \%$, and $39 \%$ in the halftherapeutic, therapeutic, and double therapeutic dose groups. Plasma creatinine level also increased significantly by $46 \%$, $78 \%$, and $137 \%$, respectively, in the MXF-treated animals.

\subsection{Influence of MXF on Plasma Biomarkers of Hepatotoxicity} in Rat. The effect of MXF on biomarkers of hepatotoxicity in rat is presented in Table 2. The plasma level of total bilirubin was significantly increased in the MXF-treated animals by $50 \%, 108 \%$, and $133 \%$ compared to control. In a similar manner, activities of alkaline phosphatase (ALP), aspartate aminotransferase (AST), and alanine aminotransferase (ALT) were significantly increased $(P<0.05)$ in the plasma of MXF-treated animals by $9 \%, 15 \%$, and $30 \% ; 19 \%, 30 \%$, and $40 \% ; 82 \%, 129 \%$, and $144 \%$, respectively, when compared to control.

3.3. Influence of MXF on Plasma Lipid Profile of Rat. Figure 2 presents the effect of MXF on plasma lipid profiles of rats following treatment with MXF. Plasma levels of total cholesterol, HDL-cholesterol, LDL-cholesterol, and triglycerides 
TABLE 2: Influence of MXF on plasma biomarkers of hepatotoxicity in rat.

\begin{tabular}{lcccc}
\hline Treatment groups & TBILI $(\mathrm{mg} / \mathrm{dL})$ & ALP $(\mathrm{U} / \mathrm{L})$ & AST (U/L) & ALT (U/L) \\
\hline CTRL & $0.12 \pm 0.02$ & $239.8 \pm 5.1$ & $62.4 \pm 2.1$ & $21.6 \pm 2.4$ \\
MXF-1 & $0.18 \pm 0.03(50 \%)^{*}$ & $262.0 \pm 4.2(9 \%)^{*}$ & $74.2 \pm 2.8(19 \%)^{*}$ & $39.4 \pm 2.7(82 \%)^{*}$ \\
MXF-2 & $0.25 \pm 0.02(108 \%)^{*}$ & $276.0 \pm 6.5(15 \%)^{*}$ & $81.0 \pm 3.7(30 \%)^{*}$ & $49.4 \pm 3.1(129 \%)^{*}$ \\
MXF-3 & $0.28 \pm 0.04(133 \%)^{*}$ & $310.6 \pm 8.3(30 \%)^{*}$ & $87.2 \pm 4.6(40 \%)^{*}$ & $52.8 \pm 3.3(144 \%)^{*}$ \\
\hline
\end{tabular}

TBILI: total bilirubin; ALP: alkaline phosphatase; AST: aspartate aminotransferase; ALT: alanine aminotransferase.

Values represent the mean \pm SD of six replicates. ${ }^{*}$ Significantly different from control $(P<0.05)$; values in parenthesis represent $\%$ of increase.

TABLE 3: Influence of MXF on the activities of hepatic antioxidant enzymes in rat.

\begin{tabular}{lccc}
\hline Treatment groups & CAT $\left(\mu \mathrm{mol} \mathrm{H}_{2} \mathrm{O}_{2}\right.$ consumed/min/mg protein) & SOD (units/mg protein) & GST (nmol/min/mg protein) \\
\hline CTRL & $0.78 \pm 0.05$ & $13.8 \pm 1.3$ & $16.3 \pm 1.7$ \\
MXF-1 & $0.64 \pm 0.07(18 \%)$ & $10.2 \pm 0.8(26 \%)^{*}$ & $14.6 \pm 1.1(10 \%)$ \\
MXF-2 & $0.53 \pm 0.03(32 \%)$ & $8.1 \pm 1.1(41 \%)^{*}$ & $12.5 \pm 0.8(23 \%)$ \\
MXF-3 & $0.44 \pm 0.02(43 \%)$ & $5.5 \pm 0.7(60 \%)^{*}$ & $10.1 \pm 0.7(38 \%)$ \\
\hline
\end{tabular}

Values represent the mean \pm SD of six replicates. ${ }^{*}$ Significantly different from control $(P<0.05)$; values in parenthesis represent $\%$ of decrease.

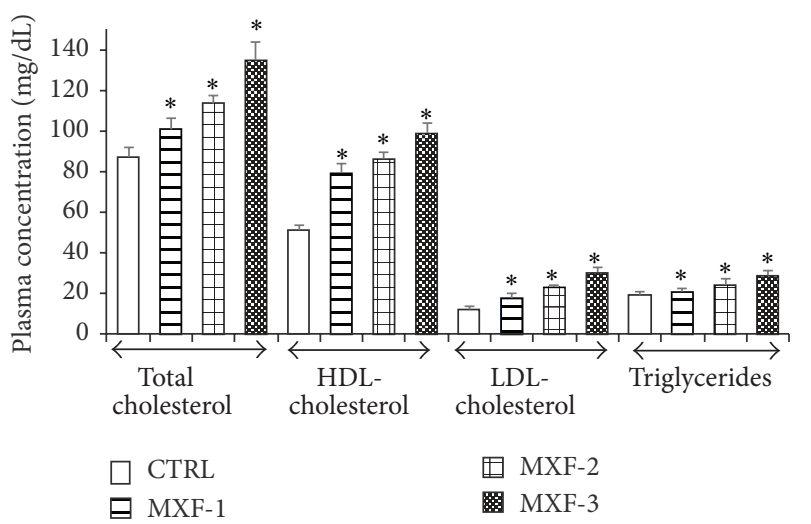

Figure 2: Influence of MXF on plasma lipid profile of rat. Values represent the mean $\pm \mathrm{SD}$ of six replicates. ${ }^{*}$ Significantly different from control $(P<0.05)$.

increased significantly $(P<0.05)$ by $16 \%, 31 \%$, and $55 \%$; $54 \%$, $68 \%$, and $92 \% ; 8 \%, 27 \%$, and $50 \%$; and $9 \%, 27 \%$, and $54 \%$, respectively, compared to control group.

3.4. Effect of MXF on the Hepatic Antioxidant Enzymes in Rat. Table 3 shows the effect of MXF for seven days on the activities of hepatic antioxidant enzymes of rat. There was a significant reduction in the activities of catalase, superoxide dismutase, and glutathione-S-transferase in the subtherapeutic, therapeutic, and double therapeutic groups by $18 \%, 32 \%$, and $43 \% ; 26 \%, 41 \%$, and $60 \%$; and $10 \%, 23 \%$, and $38 \%$, respectively, relative to control $(P<0.05)$.

\subsection{Effect of MXF on the Hepatic Nonenzymatic Antioxidants} in Rat. The effects of seven-day MXF treatment of rats on hepatic nonenzymatic antioxidants, ascorbic acid, and reduced glutathione are shown in Figures 3(a) and 3(b), respectively. The levels of the ascorbic acid and GSH were significantly reduced $(P<0.05)$ by $25 \%, 40 \%$, and $51 \%$ and $23 \%, 40 \%$, and $51 \%$, respectively, in the subtherapeutic, therapeutic, and double therapeutic MXF dose groups.

3.6. Effect of MXF on Hepatic Level of Lipid Peroxidation in Rat. The hepatic level of lipid peroxidation in rats following treatment with MXF is shown in Figure 4. Lipid peroxidation (MDA) level was significantly increased $(P<0.05)$ by $48 \%$, $58 \%$, and $74 \%$ in the liver of rats in the subtherapeutic, therapeutic, and double therapeutic MXF dose groups when compared to the control.

\section{Discussion}

In this study, we investigated the potential effect of three doses of moxifloxacin (MXF) on biomarkers of hepatotoxicity, nephrotoxicity, and oxidative stress in rat models. MXF is a fluoroquinolone antimicrobial agent with in vitro and in vivo activities against wide spectrum of Gram-negative and Gram-positive organisms [35]. Fluoroquinolones are the most widely used antibacterial agents today, thus prompting extensive studies of their potential adverse reactions in tissues in vivo. The plasma creatinine and urea levels have been widely used as suitable indicators of renal function in human and animal models [36, 37]. Creatinine is a breakdown product of creatine phosphate in muscle, while urea is major nitrogenous end product of protein and amino acid catabolism, produced by liver [38]. Data from this study suggest that MXF induced marked increase in plasma urea and creatinine levels. Creatinine and urea elevation of the plasma is an indication of abnormal renal function [39]. Plasma urea has been reported to increase in acute and chronic renal dysfunction and creatinine has been implicated in diseases such as acute kidney injury [39]. The observed increase in plasma urea and creatinine corroborates previous 


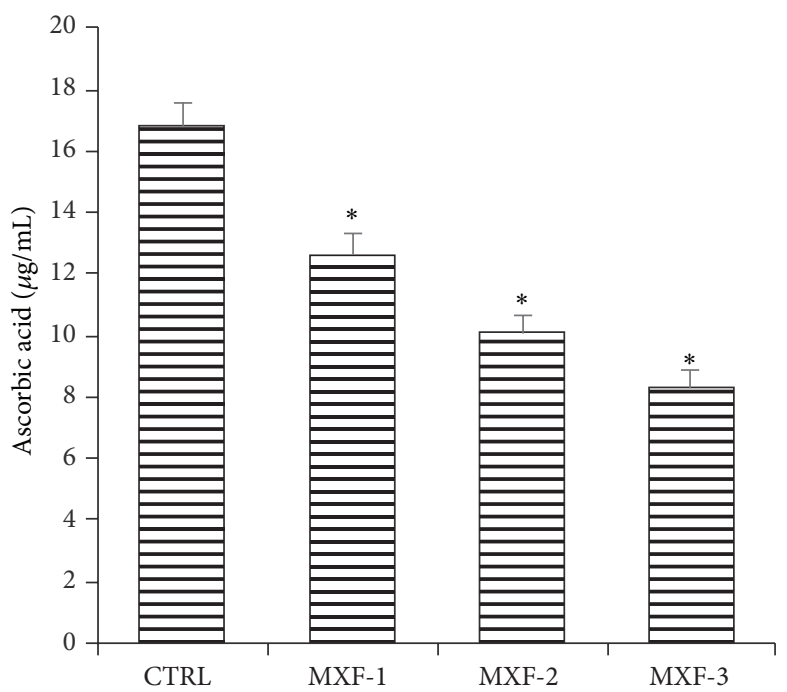

(a)

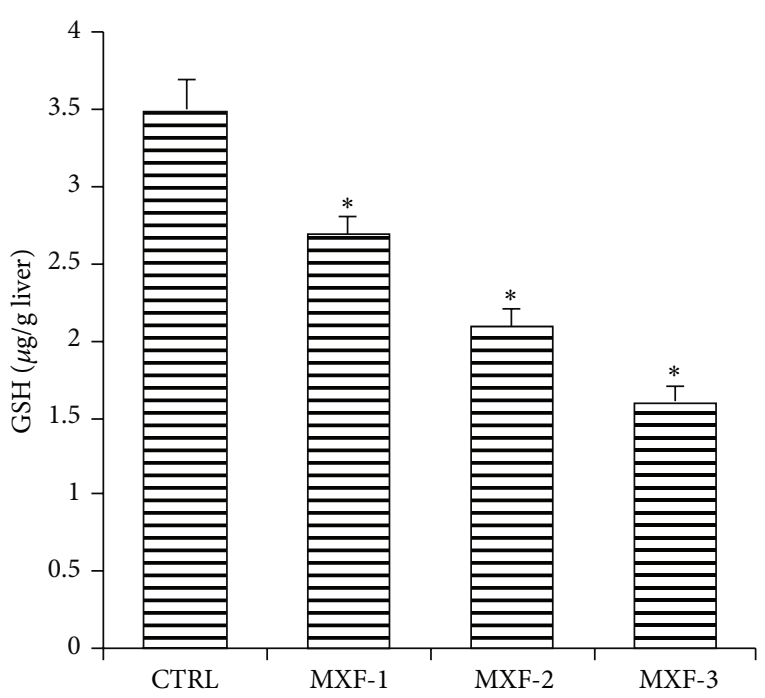

(b)

FIGURE 3: Influence of MXF on hepatic level of nonenzymatic antioxidants (a) ascorbic acid and (b) reduced glutathione levels in rat. Values represent the mean \pm SD of six replicates. * Significantly different from control $(P<0.05)$.

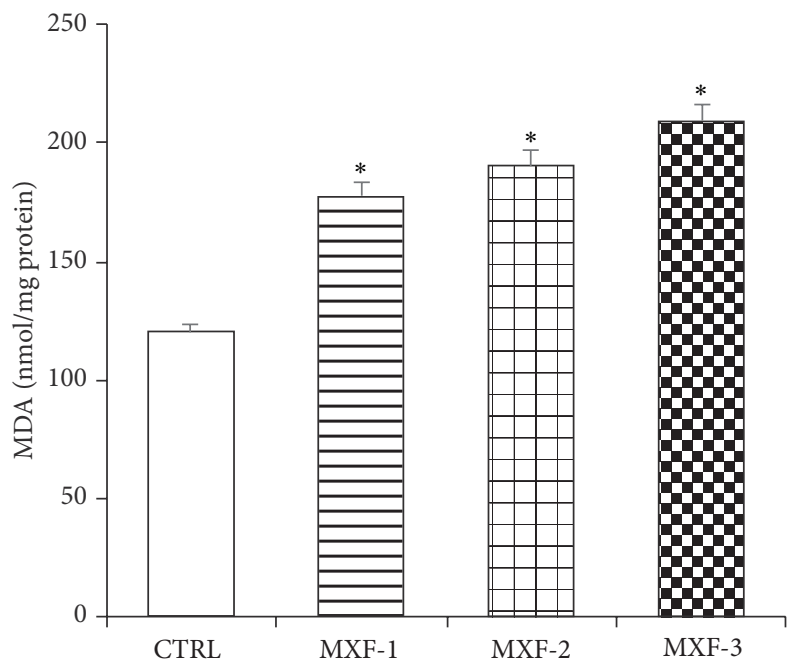

FIGURE 4: Influence of MXF on the hepatic malondialdehyde (MDA) level. Values represent the mean \pm SD of six replicates. ${ }^{*}$ Significantly different from control $(P<0.05)$.

findings following administration of fluoroquinolone antibiotics $[12,40]$.

Our results indicate that administration of MXF for seven days resulted in elevated plasma total bilirubin (TBILI) and activities of ALP, ALT, and AST in experimental animals. Increase in plasma TBILI and ALP activity is known to be associated with hepatobiliary dysfunction which may have resulted from hepatobiliary injury and cholestasis [41]. Data from this study suggests that the dose dependent increase in plasma TBILI and ALP activity caused by the doses of MXF is an indication of hepatobiliary damage. Similar observation was reported by Fatai et al. [42] from studies on a fluoroquinolone antibiotic. Activities of ALT and AST are accepted marker of hepatocellular injury in human and animal models [43]. Elevated plasma ALT and AST may be linked with membrane leakage of the hepatocyte cytosolic contents which is reflected in significant elevation of the plasma of rats treated with different doses of MXF. This observation is in consonance with previous findings on other fluoroquinolones $[44,45]$.

As a physiological mechanism to prevent injury from reactive oxygen species (ROS), cells have developed strong antioxidant defence systems. Besides scavenger molecules such as glutathione (GSH), ascorbic acid (AA), or $\alpha$ tocopherol, specific antioxidants enzymes such as catalase (CAT), superoxide dismutase (SOD), glutathione-Stransferase (GST), and glutathione peroxidase (GPx) also fulfil this task [46]. The activity or expression of these enzymes is known to be modulated by oxidative stress in vivo [47]. Data from this study indicate that the three doses of MXF administered altered both enzymatic and nonenzymatic antioxidant systems. The antioxidant enzymes CAT and SOD are part of the primary intracellular antioxidants defence mechanism against oxidative stress [47].

Superoxide radicals undergo dismutation by the action of SOD to hydrogen peroxide, while hydrogen peroxide formed is decomposed to water and molecular oxygen by CAT to prevent accumulation in the cell [48]. The decreased activities of SOD and CAT in the liver of the animals treated with the different doses of MXF could be due to the organ's response to an increased production of reactive oxygen species, as a result of exposure to the drugs and their metabolites. GST is a multifunctional enzyme and one of the key enzymes in drug metabolism, which is also known to play a vital role in redox balance in the cell [49]. It is involved in the biotransformation of xenobiotics, including drug detoxification leading to the elimination of toxic compounds [49]. The bactericidal action 
of quinolones is known to promote the generation of radicals most notably hydroxyl radicals in both Gram-negative and Gram-positive organisms as end product of drug metabolism $[50,51]$. Furthermore, studies have demonstrated the capacity of quinolone drugs to generate free radicals and alter the activities of antioxidant enzymes in vivo $[8,52]$.

The hepatic level of GSH and AA is a measure of nonenzymatic antioxidant and cellular redox status of cells [48]. Studies have shown that the redox state of intracellular AA is closely influenced by the intracellular level of GSH [53]. Our studies showed that administration of MXF causes a depression of the overall redox status in the liver as indicated by data for AA and GSH. The observed reduction in hepatic GSH and AA content is in agreement with previous findings on fluoroquinolones $[8,42,52]$.

Furthermore, data from this study indicated an alteration in levels of plasma total cholesterol, HDL-cholesterol, LDLcholesterol, and triglycerides, with a concomitant increase in the hepatic malondialdehyde (MDA) level in the MXFtreated rats. The lipoproteins are the major transporters of both cholesterol and fatty acids in vivo. Low-density lipoprotein (LDL) is a vehicle to supply cholesterol all over the body in order to maintain cell viability and to provide cholesterol for various biosynthetic processes requiring cholesterol [54] while high density lipoprotein (HDL) plays a part in reverse cholesterol transport and also protects LDL from oxidation [55]. Lipoproteins and specifically the low-density lipoprotein (LDL) particles are susceptible to oxidation and peroxidation by prooxidants. Cholesterol and polyunsaturated fatty acids (PUFA) are the main components of LDL. Oxidation of lipoproteins is a lipid peroxidation process in which the unsaturated fatty acids (PUFAS) contents are transformed into lipid hydroperoxides, MDA, and other lipid peroxidation products [56]. Therefore, the more the amount of unsaturated lipids, the greater the level of lipid peroxidation and lipid peroxidation products formed. Increase in the level of MDA is a well-established biomarker of tissue damage [57]. The high level of lipid peroxidation following the administration of doses of MXF in the liver is a characteristic feature of certain fluoroquinolones [40,44]. Depletion of the cell membrane antioxidant system is known to predispose membrane lipids to oxidation leading to accumulation of LPO products [58] including MDA as observed in this study. Previous studies have reported fluoroquinolone-induced increase in tissue MDA level [45, 59-61] and lipid peroxides [62] in vivo.

\section{Conclusion}

In conclusion, our results suggest that moxifloxacin altered renal and hepatic function as well as hepatic antioxidant defense systems of rat.

\section{Conflict of Interests}

The authors declare that there is no conflict of interests regarding the publication of this paper.

\section{References}

[1] P. M. Tulkens, P. Arvis, and F. Kruesmann, "Moxifloxacin safety: an analysis of 14 years of clinical data," Drugs in $R$ \& D, vol. 12, no. 2, pp. 71-100, 2012.

[2] P. M. Dandagi, A. M. Belekar, V. S. Mastiholimath, A. P. Gadad, V. W. Sontake, and P. S. Salian, "An improvement of the efficacy of moxifloxacin $\mathrm{HCl}$ for the treatment of bacterial keratitis by the formulation of ocular mucoadhesive microspheres," Scientia Pharmaceutica, vol. 81, no. 1, pp. 259-280, 2013.

[3] I. Kuzman, A. Bezlepko, I. K. Topuzovska et al., "Efficacy and safety of moxifloxacin in community acquired pneumonia: a prospective, multicenter, observational study (CAPRIVI)," BMC Pulmonary Medicine, vol. 14, article 105, 2014.

[4] Bayer Inc, "Moxifloxacin hydrochloride tablets," in AVELOX Product Monograph, Bayer Inc, Ontario, Canada, 2012.

[5] P. A. Moise, M. C. Birmingham, and J. J. Schentag, "Pharmacokinetics and metabolism of moxifloxacin," Drugs of Today, vol. 36, no. 4, pp. 229-244, 2000.

[6] L. D. Saravolatz and J. Leggett, "Gatifloxacin, gemifloxacin, and moxifloxacin: the role of 3 newer fluoroquinolones," Clinical Infectious Diseases, vol. 37, no. 9, pp. 1210-1215, 2003.

[7] D. N. Fish, "Fluoroquinolone adverse effects and drug interactions," Pharmacotherapy, vol. 21, no. 10, pp. 253S-272S, 2001.

[8] A. Gürbay, B. Gonthier, D. Daveloose, A. Favier, and F. Hincal, "Microsomal metabolism of ciprofloxacin generates free radicals," Free Radical Biology and Medicine, vol. 30, no. 10, pp. 11181121, 2001.

[9] S. Kubo, T. Matsumoto, K. Takahashi et al., "Enhanced chemiluminescence response of polymorphonuclear leukocytes by new quinolone antimicrobials," Chemotherapy, vol. 40, no. 5, pp. 333-336, 1994.

[10] T. Matsumoto, K. Takahashi, T. Nagafuji et al., "Fleroxacin enhancement of superoxide production by polymorphonuclear leukocytes: the role of protein kinases," Chemotherapy, vol. 42, no. 4, pp. 280-285, 1996.

[11] M. Thuong-Guyot, O. Domarle, J.-J. Pocidalo, and G. Hayem, "Effects of fluoroquinolones on cultured articular chondrocytes flow cytometric analysis of free radical production," Journal of Pharmacology and Experimental Therapeutics, vol. 271, no. 3, pp. 1544-1549, 1994.

[12] V. Sureshkumar, G. Sarathchandra, and J. Ramesh, "Biochemical, histopathological and ultra structural profile after pulsed water medication of enrofloxacin in broiler chickens," Veterinary World, vol. 6, no. 9, pp. 668-673, 2013.

[13] U. Schmidt and G. Schlüter, "Studies on the mechanism of phototoxicity of Bay y 3118 and other quinolones," in Biological Reactive Intermediates V: Basic Mechanistic Research in Toxicology and Human Risk Assessment, vol. 387 of Advances in Experimental Medicine and Biology, pp. 117-120, Springer, Berlin, Germany, 1996.

[14] E. Yazar and B. Tras, "Effects of fluoroquinolone antibiotics on hepatic superoxide dismutase and glutathione peroxidase activities in healthy and experimentally induced peritonitis mice," Revue de Médecine Vétérinaire, vol. 152, no. 3, pp. 235-238, 2001.

[15] M. Kavutcu, O. Canbolat, S. Öztürk et al., "Reduced enzymatic antioxidant defense mechanism in kidney tissues from gentamicin-treated guinea pigs: effects of vitamins $\mathrm{E}$ and C," Nephron, vol. 72, no. 2, pp. 269-274, 1996.

[16] K. Rahman, "Studies on free radicals, antioxidants, and cofactors," Clinical Interventions in Aging, vol. 2, no. 2, pp. 219-236, 2007. 
[17] I. Albesa, M. C. Becerra, P. C. Battán, and P. L. Páez, “Oxidative stress involved in the antibacterial action of different antibiotics," Biochemical and Biophysical Research Communications, vol. 317, no. 2, pp. 605-609, 2004.

[18] D. J. Dwyer, M. A. Kohanski, B. Hayete, and J. J. Collins, "Gyrase inhibitors induce an oxidative damage cellular death pathway in Escherichia coli," Molecular Systems Biology, vol. 3, article 91, 2007.

[19] F. A. D. T. G. Wagener, C. E. Carels, and D. M. S. Lundvig, "Targeting the redox balance in inflammatory skin conditions," International Journal of Molecular Sciences, vol. 14, no. 5, pp. 9126-9167, 2013.

[20] X. Wang, X. Zhao, M. Malik, and K. Drlica, "Contribution of reactive oxygen species to pathways of quinolone-mediated bacterial cell death," Journal of Antimicrobial Chemotherapy, vol. 65 , no. 3, pp. 520-524, 2010.

[21] P. Ø. Jensen, A. Briales, R. P. Brochmann et al., "Formation of hydroxyl radicals contributes to the bactericidal activity of ciprofloxacin against Pseudomonas aeruginosa biofilms," Pathogens and Disease, vol. 70, no. 3, pp. 440-443, 2014.

[22] National Research Council, Guide for the Care and Use of Laboratory Animals, The National Academies Press, National Research Council, Washington, DC, USA, 8th edition, 2011.

[23] A. G. Gornall, C. J. Bardwawill, and M. M. David, "Determination of serum proteins by means of the biuret reaction," The Journal of Biological Chemistry, vol. 177, no. 2, pp. 751-766, 1949.

[24] E. R. Jaffé, "Oxidative hemolysis, or what made the red cell break?" The New England Journal of Medicine, vol. 286, no. 3, pp. 156-157, 1972.

[25] N. W. Tietz, Clinical Guide to Laboratory Tests, W.B. Saunders, Philadelphia, Pa, USA, 3rd edition, 1995.

[26] N. W. Tietz, E. L. Pruden, and O. Siggaard-Andersen, "Electrolytes," in Tietz Textbook of Clinical Chemistry, C. A. Burtis and E. R. Ashwell, Eds., pp. 1354-1374, WB Saunders, London, UK, 1994.

[27] S. Reltman and S. A. Frankel, "Colorimetric method for the determination of serum ALT and AST," American Journal of Clinical Pathology, vol. 28, pp. 56-63, 1957.

[28] P. Trinder, "Enzymatic colorimetric method of cholesterol determination," Annals of Clinical Biochemistry, vol. 6, article 24, 1969.

[29] D. J. Jollow, J. R. Mitchell, N. Zampaglione, and J. R. Gillette, "Bromobenzene induced liver necrosis, protective role of glutathione and evidence for 3,4 bromobenzene oxide as the hepatotoxic metabolite," Pharmacology, vol. 11, no. 3, pp. 151169, 1974.

[30] S. K. Jagota and H. M. Dani, "A new colorimetric technique for the estimation of vitamin C using Folin phenol reagent," Analytical Biochemistry, vol. 127, no. 1, pp. 178-182, 1982.

[31] W. H. Habig, M. J. Pabst, and W. B. Jakoby, "Glutathione S-transferases. The first enzymatic step in mercapturic acid formation," Journal of Biological Chemistry, vol. 249, no. 22, pp. 7130-7139, 1974.

[32] H. P. Misra and I. Fridovich, "The role of superoxide anion in the auto-oxidation of epinephrine and a simple assay for superoxide dismutase," The Journal of Biological Chemistry, vol. 247, no. 10, pp. 3170-3175, 1972.

[33] A. K. Sinha, "Colorimetric assay of catalase," Analytical Biochemistry, vol. 47, no. 2, pp. 389-394, 1972.
[34] R. Varshney and R. K. Kale, "Effects of calmodulin antagonists on radiation-induced lipid peroxidation in microsomes," International Journal of Radiation Biology, vol. 58, no. 5, pp. 733-743, 1990.

[35] J.-P. Caeiro and P. B. Iannini, "Moxifloxacin (Avelox): a novel fluoroquinolone with a broad spectrum of activity," Expert Review of Anti-Infective Therapy, vol. 1, no. 3, pp. 363-370, 2003.

[36] M. A. Ferguson and S. S. Waikar, "Established and emerging markers of kidney function," Clinical Chemistry, vol. 58, no. 4, pp. 680-689, 2012.

[37] A. Simsek, V. Tugcu, and A. I. Tasci, "New biomarkers for the quick detection of acute kidney injury," ISRN Nephrology, vol. 2013, Article ID 394582, 9 pages, 2013.

[38] S. Gowda, P. B. Desai, S. S. Kulkarni, V. V. Hull, A. A. K. Math, and S. N. Vernekar, "Markers of renal function tests," North American Journal of Medical Sciences, vol. 2, no. 4, pp. 170-173, 2010.

[39] A. Akcay, K. Turkmen, D. Lee, and C. L. Edelstein, "Update on the diagnosis and management of acute kidney injury," International Journal of Nephrology and Renovascular Disease, vol. 3, pp. 129-140, 2010.

[40] E. Olayinka, A. Ore, and O. Adeyemo, "Alterations in biochemical indices and antioxidant status in rats following treatment with gatifloxacin," British Journal of Pharmaceutical Research, vol. 6, no. 5, pp. 293-305, 2015.

[41] A. Singh, T. K. Bhat, and O. P. Sharma, "Clinical biochemistry of hepatotoxicity," Journal of Clinical Toxicology, S4, article 1, 2011.

[42] I. M. Fatai, N. O. A. Imaga, and G. O. Gbenle, "Biochemical investigations into the effects of co-administration of ciprofloxacin and nicosan," African Journal of Pharmacy and Pharmacology, vol. 7, no. 39, pp. 2674-2679, 2013.

[43] D. E. Amacher, "Serum transaminase elevations as indicators of hepatic injury following the administration of drugs," Regulatory Toxicology and Pharmacology, vol. 27, no. 2, pp. 119-130, 1998.

[44] E. T. Olayinka and A. Ore, "Administration of clarithromycin (Claricin) induces changes in antioxidant status and biochemical indices in rats," Research Journal of Pharmacology, vol. 6, no. 4, pp. 52-61, 2012.

[45] E. T. Olayinka, A. Ore, and O. S. Ola, "Influence of different doses of levofloxacin on antioxidant defense systems and markers of renal and hepatic dysfunctions in rats," Advances in Toxicology, vol. 2015, Article ID 385023, 7 pages, 2015.

[46] T. P. A. Devasagayam, J. C. Tilak, K. K. Boloor, K. S. Sane, S. S. Ghaskadbi, and R. D. Lele, "Free radicals and antioxidants in human health: current status and future prospects," Journal of Association of Physicians of India, vol. 52, pp. 794-804, 2004.

[47] M. Valko, D. Leibfritz, J. Moncol, M. T. D. Cronin, M. Mazur, and J. Telser, "Free radicals and antioxidants in normal physiological functions and human disease," The International Journal of Biochemistry \& Cell Biology, vol. 39, no. 1, pp. 44-84, 2007.

[48] B. Halliwell, "Free radicals and other reactive species in disease," in Encyclopedia of Life Sciences, pp. 1-9, 2015.

[49] A. Pastore, G. Federici, E. Bertini, and F. Piemonte, "Analysis of glutathione: implication in redox and detoxification," Clinica Chimica Acta, vol. 333, no. 1-2, pp. 19-39, 2003.

[50] M. A. Kohanski, D. J. Dwyer, B. Hayete, C. A. Lawrence, and J. J. Collins, "A common mechanism of cellular death induced by bactericidal antibiotics," Cell, vol. 130, no. 5, pp. 797-810, 2007.

[51] M. A. Kohanski, D. J. Dwyer, and J. J. Collins, "How antibiotics kill bacteria: from targets to networks," Nature Reviews Microbiology, vol. 8, no. 6, pp. 423-435, 2010. 
[52] V. Sureshkumar, G. Sarathchandra, and J. Ramesh, "Veterinary pharmacovigilance evaluation on impact of enrofloxacin administration on antioxidant status in broiler chicken," International Journal of Current Microbiology and Applied Sciences, vol. 2, no. 5, pp. 335-341, 2013.

[53] A. Meister, "Glutathione-ascorbic acid antioxidant system in animals," The Journal of Biological Chemistry, vol. 269, no. 13, pp. 9397-9400, 1994.

[54] G. H. Tomkin and D. Owens, "LDL as a cause of atherosclerosis," The Open Atherosclerosis \& Thrombosis Journal, vol. 5, no. 1, pp. 13-21, 2012.

[55] A. V. Khera, M. Cuchel, M. de la Llera-Moya et al., "Cholesterol efflux capacity, high-density lipoprotein function, and atherosclerosis," The New England Journal of Medicine, vol. 364, no. 2, pp. 127-135, 2011.

[56] B.-L. Yu, S.-P. Zhao, and X.-S. Huang, "Oxidized low-density lipoprotein: a double-edged sword on atherosclerosis," Medical Hypotheses, vol. 69, no. 3, pp. 553-556, 2007.

[57] C. M. Spickett, I. Wiswedel, W. Siems, K. Zarkovic, and N. Zarkovic, "Advances in methods for the determination of biologically relevant lipid peroxidation products," Free Radical Research, vol. 44, no. 10, pp. 1172-1202, 2010.

[58] K. S. Montine, J. F. Quinn, T. J. Montine, V. Talla, and P. R. Veerareddy, "Overview: membrane lipid peroxidation," in Membrane Lipid Signaling in Aging and Age-Related Disease, vol. 12 of Advances in Cell Aging and Gastrontology, pp. 11-26, Elsevier Science \& Technology, 2003.

[59] S. M. Rawi, I. M. Mourad, N. M. S. Arafa, and N. I. Alazabi, "Effect of ciprofloxacin and levofloxacin on some oxidative stress parameters in brain regions of male albino rats," African Journal of Pharmacy and Pharmacology, vol. 5, no. 16, pp. 18881897, 2011.

[60] O. Ioannidis, B. Papaziogas, P. Tsiaousis, G. Paraskevas, E. J. Giamarellos-Bourboulis, and I. Koutelidakis, "Effect of moxifloxacin on survival, lipid peroxidation and inflammation in immunosuppressed rats with soft tissue infection caused by Stenotrophomonas maltophilia," Microbiology and Immunology, vol. 58, no. 2, pp. 96-102, 2014.

[61] E. T. Olayinka and A. Ore, "Influence of Azithromycin treatment on hepatic lipid peroxidation and antioxidant defence systems of rats," British Journal of Pharmaceutical Research, vol. 4, no. 2, pp. 240-256, 2014.

[62] V. Talla and P. R. Veerareddy, "Oxidative stress induced by fluoroquinolones on treatment for complicated urinary tract infections in Indian patients," Journal of Young Pharmacists, vol. 3, no. 4, pp. 304-309, 2011. 

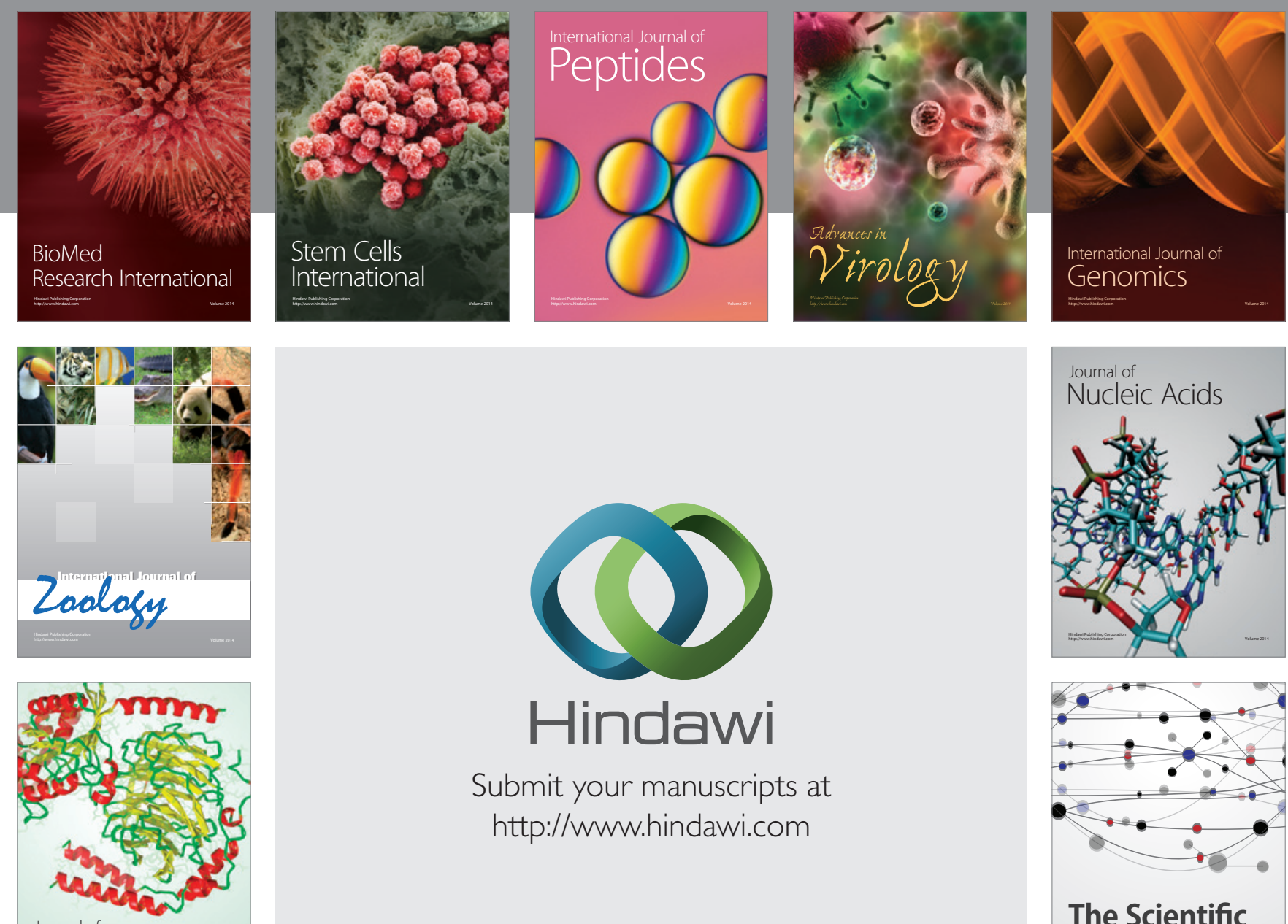

Submit your manuscripts at

http://www.hindawi.com

Journal of
Signal Transduction
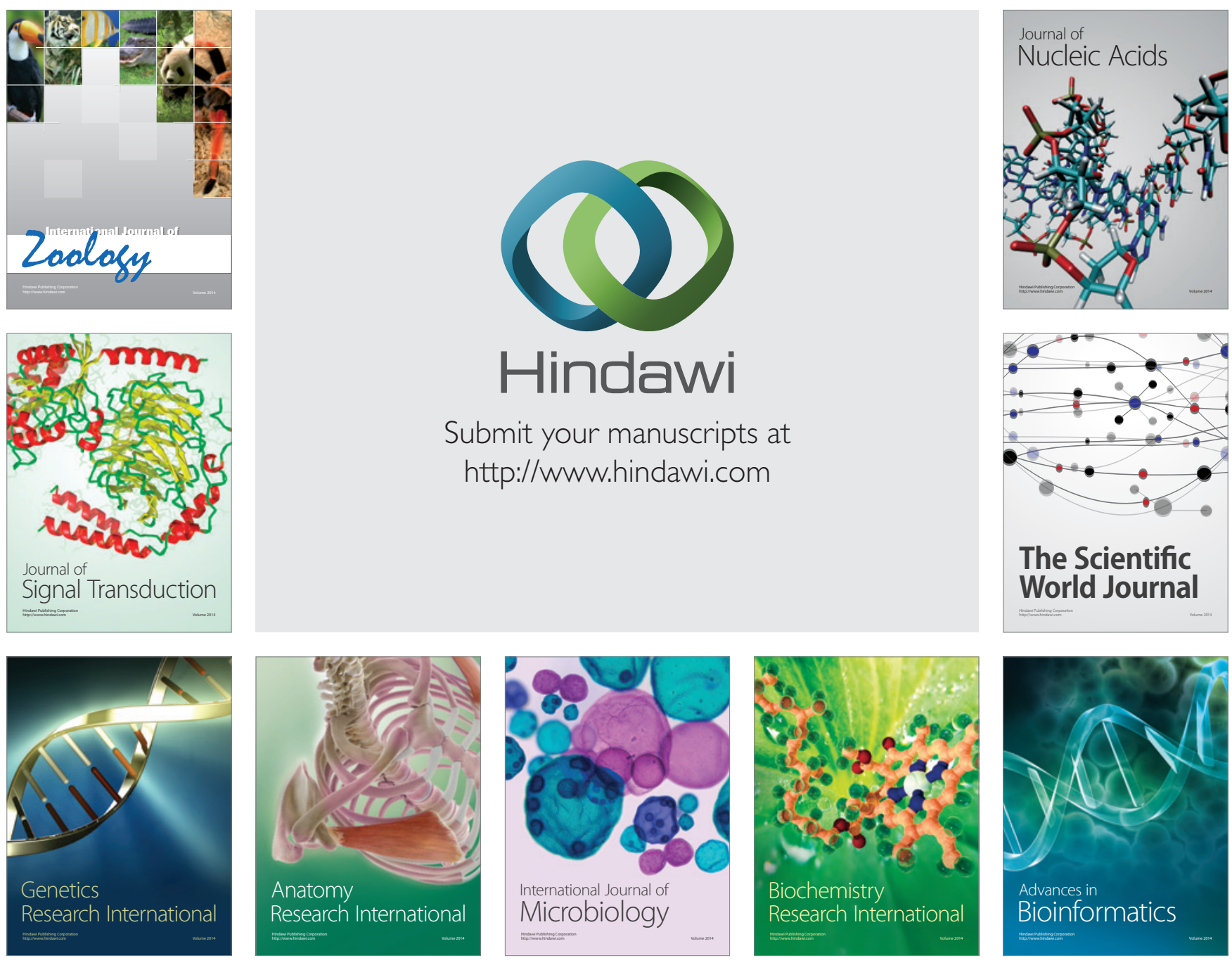

The Scientific World Journal
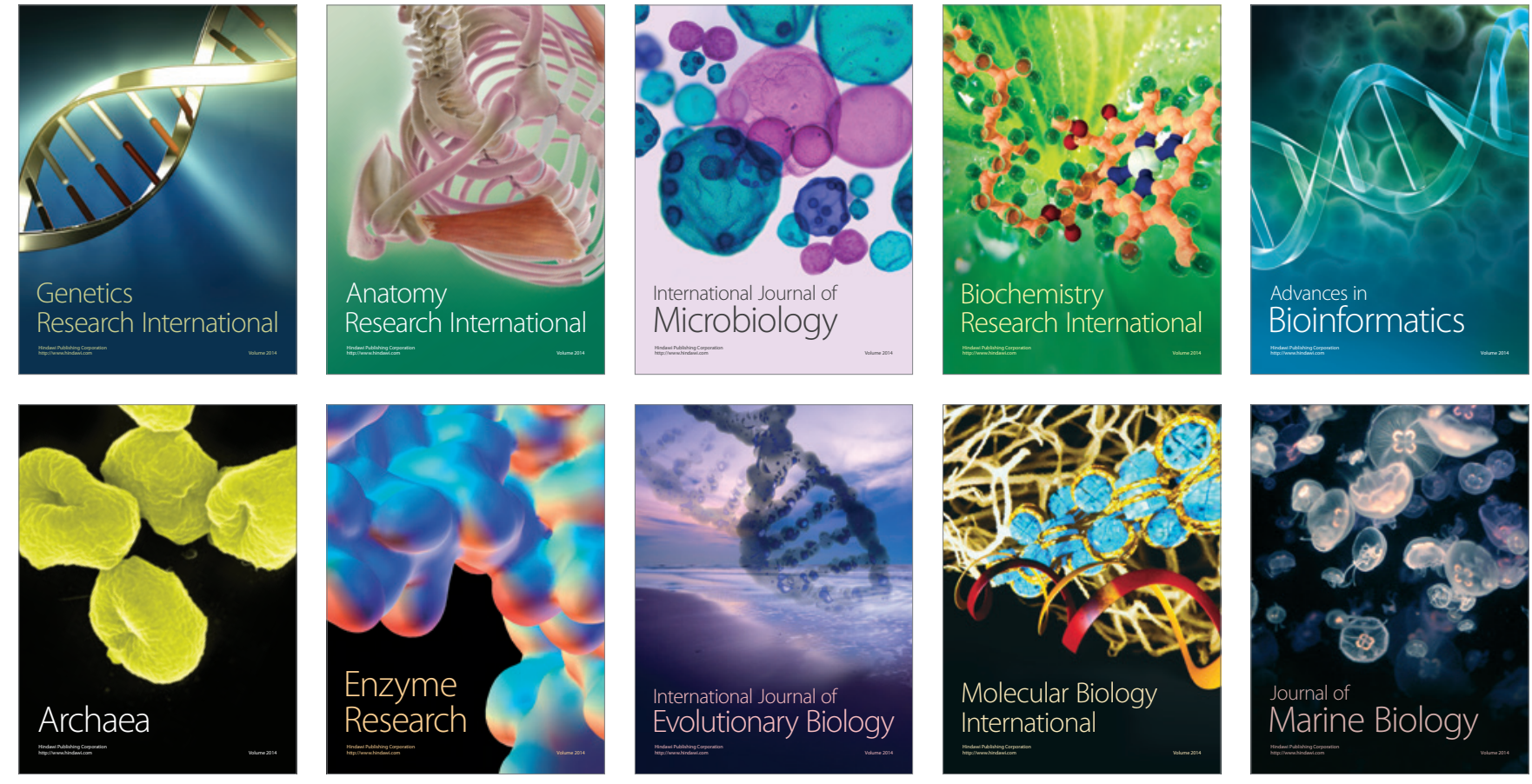